TIPA. Travaux interdisciplinaires sur la parole et le langage

31 | 2015

L'impact du contact entre les langues

\title{
L'impact du futur périphrastique français dans les franco-créoles
}

The impact of French periphrastic future on the development of French-based Creoles

Marie E. Paul

\section{OpenEdition}

Journals

Édition électronique

URL : http://journals.openedition.org/tipa/1412

DOI : 10.4000/tipa.1412

ISSN : 2264-7082

Éditeur

Laboratoire Parole et Langage

Référence électronique

Marie E. Paul, «L'impact du futur périphrastique français dans les franco-créoles », TIPA. Travaux interdisciplinaires sur la parole et le langage [En ligne], 31 | 2015, mis en ligne le 22 décembre 2015, consulté le 26 mai 2020. URL : http://journals.openedition.org/tipa/1412 ; DOI : https://doi.org/ 10.4000/tipa. 1412

Ce document a été généré automatiquement le 26 mai 2020

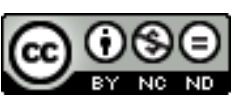

La revue TIPA. Travaux interdisciplinaires sur la parole et le langage est mise à disposition selon les termes de la licence Creative Commons Attribution - Pas d'Utilisation Commerciale - Pas de Modification 4.0 International. 


\title{
L'impact du futur périphrastique français dans les franco-créoles
}

\author{
The impact of French periphrastic future on the development of French-based \\ Creoles
}

Marie E. Paul

\section{Introduction}

1 S. G. Thomason (2001) définit le contact linguistique comme suit : « Language contact is the use of more than one language in the same place at the same time (...) in situations in which at least some people use more than one language ». Il s'agit donc d'une situation où plusieurs langues sont en usage dans un même espace et au même moment de telle sorte qu'au moins une partie des personnes fait usage de plus d'une langue. Dans la réédition de 1952 des Langues du monde, les langues créoles sont traitées à deux niveaux. Elles apparaissent premièrement, dans la section des langues indoeuropéennes, à la suite des parlers romans. Elles sont traitées par J. Vendryes et E. Benveniste ${ }^{1}$ comme des langues issues du contact de langues romanes et de langues indigènes ou importées : "Sur plusieurs points du globe, le portugais, l'espagnol et le français, en contact avec des langues indigènes ou importées (Antilles), ont produit divers parlers créoles... ». Ainsi, compte tenu de ces définitions, les langues créoles peuvent être considérées comme des langues issues de contacts sans que cette définition leur soit exclusive.

Bien au contraire, si l'on tient compte de l'histoire des langues romanes Goyette (2000) estime qu'il y a matière à établir de sérieuses comparaisons. Compte tenu du contexte de conquête, de déplacement de population et d'esclavage où l'éducation formelle n'était accessible qu'à l'élite, Goyette stipule qu'il n'y a pas de raison d'exclure la possibilité que le latin ait connu une certaine créolisation lorsque l'on compare son histoire externe à celle d'autres langues européennes. Ainsi, il avance l'hypothèse, que le proto-roman est né du contact de variétés créolisées et non-créolisées de latin: «Early romance was originally born out of the contact of creolized and non-creolized 
varieties of Latin » (2000:4). Il précise ensuite qu'il veut distinguer le phénomène du contact dans les langues romanes (phénomène qu'il estime bien documenté sur notamment le rôle des influences du gaulois et du franc sur le latin) du phénomène de la créolisation qui, selon lui, a de toute vraisemblance joué un rôle dans l'histoire des langues romanes plus que dans d'autres langues².

3 Adam (1883) émet l'hypothèse que le passage du type synthétique au type analytique observé dans les langues européennes serait dû à cette situation de contact lors de la transmission de la langue par les vainqueurs. Il prend en exemple le passage du latin aux langues romanes mais considère que le phénomène s'applique à l'ensemble des langues indo-européennes désignées comme des langues aryennes :

Il est dans la science un problème qui n'a point été résolu, je veux parler du passage de toutes les langues aryennes anciennes de l'état synthétique à l'état analytique, évolution qui contraste singulièrement avec la fixité des langues sémitiques. Or, les tribus originaires du plateau de Pamir ont successivement émigré dans toutes les directions, s'implantant au milieu de populations anaryennes qui parlaient d'autres langues, les soumettant par la force de leurs armes, et à la longue, se les assimilant. N'est-il pas fondé de se demander si le passage de la synthèse à l'analyse n'a point eu pour cause, partiellement au moins, l'effort instinctif des vaincus qui, ayant adopté la langue des vainqueurs, ont maintenu leur grammaire propre dans des patois qui ne nous sont point parvenus, dans des parlers ruraux analogues à ce latin rustique d'où les langues romanes sont issues ? [...] Est-il certain que ces descendants de vaincus, plus ou moins assimilés par les vainqueurs, n'avaient point conservé, dans les provincialismes de leur méchant latin quelques traces grammaticales des idiomes anaryens parlés par leurs ancêtres?

Le même constat de contact linguistique et de changements vers des formes plutôt analytiques a été établi par Schleicher (1852: 24-25) au sujet des langues indoeuropéennes: «N'oubliez pas non plus que toutes ces nations, les véritables pionniers de la civilisation humaine, se sont mises en contact permanent entre elles ; c'est encore là un motif, du moins accessoire, de la décroissance des idiomes primitifs ». Bien plus, il va jusqu'à identifier d'autres éléments en cause dans le changement linguistique qu'il désigne malheureusement du terme péjoratif de "dégénérescence »: "Les grandes époques, ce qu'on pourrait appeler les cataclysmes des races et des sociétés, sont accompagnées d'une rapide décroissance des idiomes, la migration des peuples vers l'empire romain était suivie d'une dégénérescence subite des langues romanes et germaniques».

R. Chaudenson (2006) a souligné, aussi, ce fait dans le compte rendu des actes du colloque 'Créolisation linguistique et sciences humaines' traitant du caractère plutôt analytique ou synthétique de l'expression du futur dans la lignée des langues indoeuropéennes. En somme, il faut comprendre que le phénomène dont il est question, à savoir le passage du "type synthétique» à "l'analytique», correspond à un phénomène généralement observé dans les langues du monde.

Dans la littérature spécialisée, la filiation des langues créoles fait débat. Leur statut de langues issues de contact fait qu'elles sont d'ordinaire désignées comme une catégorie à part ou atypique (Thomason \& Kaufman 1988, I. Dyen, J. Kruskal \& P. Black 1992). 
D'autres, considèrent la naissance de ces langues comme non-exceptionnelle (Michel Degraff 2003, Mufwene 2007). Au nombre des éléments présentés pour avancer le classement des langues créoles dans 'une catégorie à part' sont cités les marqueurs préverbaux. Ils sont perçus comme comme faisant partie des éléments les plus divergents de la langue européenne, de par leurs formes actuelles ainsi que leur fonctionnement plutôt analytique. Pourtant, et nous allons essayer de le montrer, le mode d'expression du futur dans les créoles français est indubitablement d'origine française. Elle se confirme à travers l'étude des formes, du sémantisme, et du fonctionnement syntaxique.

7 Nous entendons montrer grâce à l'exploitation d'un corpus ${ }^{3}$ composé de textes faisant montre de variétés de français colonial mais aussi de textes créoles anciens en antillais, haïtien et louisianais datés respectivement de 1671 à 1850,1804 et 1867, l'impact des variétés de français colonial sur le développement du futur dans les franco-créoles. Nous procèderons comme suit : nous essaierons dans un premier temps de comprendre comment et pourquoi le futur périphrastique a été préféré par les colons. Puis nous présenterons les résultats de l'analyse des textes français suivis de ceux de l'analyse des textes créoles. Enfin nous mettrons en évidence les correspondances de formes entre les deux ensembles.

\section{Comment et pourquoi le futur périphrastique a-t-il été préféré au futur synthétique?}

8 Le futur périphrastique présente le procès comme imminent donc assuré alors que le futur simple peut difficilement exprimer cette immédiateté. Quoiqu'elle n'ait pas exactement le même sens que le futur simple rendu par la périphrase verbale avec aller s'est révélé un sérieux concurrent au futur simple. La préférence pour la périphrase verbale (aller+infinitif) s'explique par les difficultés engendrées par le manque d'analogie du futur synthétique mais également parce que les formes analytiques, dit Meillet (1948), permettent une meilleure expressivité.

9 T.G. Fennell, dans son ouvrage La morphologie du futur en moyen français (1975), étudie des textes populaires (farces, sotties) et des textes versifiés datés de 1350 à 1650 afin de mettre en évidence les évolutions du futur simple en moyen français. Il affirme que la morphologie du futur manque de clarté et d'universalité ce qui constitue, selon lui, une faiblesse analogique car «la solidité d'une création morphologique réside en partie dans sa régularité et dans la constance de sa formation (p. 4) ». Quelques lignes plus bas, l'auteur établit un parallèle avec le subjonctif présent considérant la multiplicité des formes, il déclare que «Cet état de choses peut mener à la confusion, et se prête facilement aux aberrations » et que les nombreuses homonymies du futur de l'époque ainsi que la difficulté due aux irrégularités étaient fâcheuses. Fennell interroge son public et demande : «Quel est aujourd'hui le futur de bouillir ou de choir? » avant de répondre «La rareté de ces formes fait hésiter le sujet parlant, et il choisit une autre tournure ". Rappelons-le, nous cherchons à comprendre ici l'histoire, l'évolution du marqueur du futur dans les créoles français qui nous intéressent. Si Fennell (1975) convient de la rareté et des irrégularités du futur simple du moyen français, qu'en dire des français ordinaires qui ont colonisé la zone américano-caraïbe ? Alors qu'en France métropolitaine les grammairiens contribuaient à normer la langue, il est fort probable que les effets de cette normalisation aient été moindres Outre-mer puisque, à 
considérer l'ensemble des variétés du français colonial, c'est la tournure périphrastique qui est préférée et utilisée par ces émigrés français.

Lars-Göran Sundell (1991), dans Le temps futur en français moderne, précise que le futur périphrastique quoique attesté depuis le XIII ème siècle dans Perceval ou la quête du Saint Graal n'aura véritablement droit de cité qu'au XV ${ }^{\text {ème }}$ siècle. R. Martin et M. Wilmet (1980) écrivent qu'il prend son essor au XVe siècle "sans qu'on ose affirmer qu'il appartient dès ce moment à la norme du moyen français ». Ces explications aident à comprendre le faible taux d'utilisation du futur périphrastique comparativement au futur simple dans l'étude de Sundell. Cette étude expose un fait intéressant: quoique travaillant sur le français contemporain, ces résultats peuvent également apporter un éclairage pour les locuteurs du français colonial. Sur 2573 cas de futur simple et 1575 cas de futur périphrastique, l'étude montre (p.20) que le pourcentage de futur périphrastique est supérieur au futur simple pour les première et deuxième personnes du singulier soit $34 \%$ opposé à $22 \%$ pour la $1^{\text {ère }}$ personne et $14 \%$ opposé à $10 \%$ pour la deuxième du singulier.

11 Ces études montrent que, d'une part, le futur simple français (d'après des documents datés de 1350 à 1650) manque de régularité dans sa formation. Ceci a pour conséquence que les locuteurs tendent à changer de construction pour éviter les écueils. Bauche (1946) fait le même constat. D'après Bauche, le futur traverse une crise en LP (Langage populaire), le futur selon la formation romane est en régression au profit du futur de forme germanique, formé avec un auxiliaire, explique-t-il. Toujours selon Bauche (1946), le futur antérieur semble être préféré au futur simple, le choix entre l'auxiliaire être ou avoir est parfois inversé par rapport au français standard. Toutefois, entre le futur simple, 'synthétique', le futur périphrastique et le futur antérieur, le plus usité est le futur périphrastique. Dans tous les cas il insiste sur le fait que le futur synthétique s'emploie peu en LP. D'autre part si le futur périphrastique est attesté depuis le XIII ${ }^{\text {ème }}$ siècle, il n'a pas droit de cité dans la grammaire tandis qu'aux première et deuxième personnes du sujet (embrayeurs personnels de la situation d'énonciation) son pourcentage d'utilisation dépasse celui du futur simple.

Dans ce travail, nous traitons davantage du futur analytique que du futur synthétique puisque ce dernier tend à être délaissé au profit du premier. Voyons maintenant ce que révèle l'étude des variétés de français colonial tel que parlé à Saint-Barthélémy, dans le Missouri, à Frenchville, au Canada et en Louisiane.

\section{Aller + infinitif dans les variétés de français colonial}

En Louisiane, selon Eveline Pellerin (1937), le franco-louisianais s'exprime au «[...] futur en se servant du présent du verbe aller et d'un infinitif ». Elle explique qu'il fait davantage usage d'un 'j'vas aller' au lieu de 'j'irai', ou 'il va finir' pour 'il finira' ou encore 'nous allons venir' au lieu de 'nous viendrons'. Elle cite également pour exemple la structure (je m'en vais + infinitif), elle semble plus rare et serait utilisée à la première personne du singulier et de préférence par les anciens. Elle déclare aussi que «si le Louisianais se sert du futur, pour la plupart du temps, ce sera un futur incorrect, soit de prononciation, soit de forme ".

Nous constatons également après l'étude des contes du Missouri et du «Survival of French » que le futur synthétique est excessivement rare, le futur périphrastique est préféré. Un comptage opéré sur les 13 contes (Médard Carrière) susmentionnés révèle 
que le futur périphrastique est toujours utilisé majoritairement par rapport au futur synthétique. Lorsqu'il n'est pas utilisé de manière exclusive dans un conte, le nombre d'emplois de futur périphrastique peut être deux ou trois fois supérieur au futur synthétique. Dans la catégorie du futur périphrastique la forme en va est majoritaire. On la rencontre à la première, deuxième et troisième personne du singulier, comme en témoignent les exemples cités ci-dessous. La forme du futur périphrastique élidée en $a$ est observée dans des contextes où la forme forte du pronom personnel sujet (moin, suivi de la reprise $m$ ), ou $t$ ' la précède.

Selon Dörper (1990), $m a+$ inf. est également une construction courante dans le français régional du Québec où la forme n'est en règle générale pas niée. Il est fait usage de m'a $y$ aller ou j'irai pas à la forme négative. Sur 284 attestations de variantes de "s'en aller + inf. ». dans Le Trésor de la langue française au Québec, 133 sont réalisées en ma + inf. Ce chiffre témoigne du fort taux d'utilisation de cette forme courte dans un français « colonial » comme celui du Québec.

16 Notre analyse de Théâtre 1 de Michel Tremblay (1991) conforte les observations de Dörper (1990). En effet, nous avons pu relever plusieurs occurrences de futur périphrastique dans ce texte caractéristique du joual. Des attestations de futur en $v a$ et ma sont relevées :

p. 403 : Elle, a'va me payer ça!;

p. 259 : J'pense que j'vas aller jeter un coup d'œil ;

p. 253 : Dis-moé pourquoi c' que t'as peur de lui peur, pis m'as te dire pourquoi c'qu'y vient m'écœurer ;

p. 251 : la nouvelle court qu'à soir va être un grand soir ;

p. 93 : pis ma tante a'va toute te faire ça ;

p. 93 : pis a'va en remettre à part de ça, elle ! ;

p. 83 : m'as t'en donner, pis gratis.

La préférence pour cette forme courte du futur périphrastique est attestée dans bien d'autres variétés de français Outre-mer.

Les exemples suivants proviennent du français du Missouri. Ces attestations de futur périphrastique sont tirées du conte numéro 2 ( $\mathrm{C} 2$, Médard Carrière). Notez la forme contractée de vas en a notamment à la première ou deuxième personne du singulier :

19 Je m'en vas par derrière, pis quand m'as l'attraper, je l'mange. L' loup i' dzit: «M'as aller avec touè pis m'as t'aider ». (C53, p. 246) :j'vas t' la donner, pis le guiab' va v'nir t' qu'ri par rapporte $q u$ ' tsu vas avouère sept ans et de Ward Allison Dorrance : p. $114:$ Ma te donner eunne boule de laine. Tu pourras la 'marrer après ta garlite..., W.A.D.115: "Quoi c'est on va faire?", "Belle de Jour a'va vous pagner tous les jours. Belle Finette a'va balier. Et mouè, ma faire à manger », W.A.D.115: « quand t'es après pagner la vieille, ma t'apporter la hache, pi tu pourras lui couper son cou ", W.A.D.115 : ta voler mon cheval, pi tu pourras aouère ma fille en mariage, 116 : «mouè ma cogner!», $118:$ Ben , ma 'marrer mon boeu. Ma aller kri l'aut.

Il semble, aussi, toujours d'après les contes du Missouri et "The Survival of French ", que le futur périphrastique apparaisse dans sa forme contractée davantage à la première personne du singulier. Le conte 62 p. 279 (Médard Carrière) montre plusieurs occurrences de futur contracté à la première personne et de quelques cas, certes beaucoup plus rares, à la deuxième personne du singulier : en voici quelques extraits 'quand les rabioles 'l ont été murtes, Saint Michel "l a d'mandé au guiab": 'quocé t'as prend', la tête ou ben les racines?' L' guiab' 'l a dzit i aurait pris les têtes .... ; Saint-Michel 'l a dzit au 
guiab' : quocé t'as prend c'coup-là, les têtes ou les racines ?'-'m'as pren les racines,' i'dzit 'c'coup là '(C 25 p. 124) t'as tout fouillé la mur de pierre.

Il se trouve en effet, à bien considérer les exemples précédents, que le passé composé et le futur peuvent être homophones. Si l'on observe le dernier exemple de la série, hormis le contexte, t'as fouillé peut être un futur périphrastique ou un passé composé avec l'auxiliaire avoir. L'auxiliaire du passé composé et du futur a encore un autre homophone.

22 En ce qui concerne le français de Saint Barthélemy, selon G. Lefebvre (1976: 139) le futur éloigné est rendu par le verbe aller conjugué à toutes les personnes + verbe. Soit les exemples suivants :

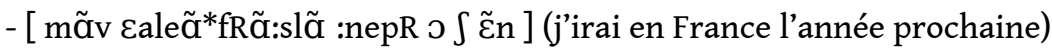

- [ ty va aleã *fRã:slã :nepR $0 \int \tilde{\varepsilon} n$ ] (tu iras en France l'année prochaine)

- [ כ̃ va ale õ *fRã:slã :nepR $0 \int \tilde{\varepsilon} n$ ] (nous irons en France l'année prochaine).

A ce propos, Lefebvre note une absence quasi complète de la conjugaison avec 'nous' dans cette variété de français.

L'utilisation de aller + verbe Inf. est confirmée dans Chaudenson \& Calvet (1998) dans

l'exemple $\mathrm{n}^{\circ} 14$ : quand tu seras adultes, tu feras la même chose $=>$ 'Amik tu sra gran tu va fèr la mèm choz' mais on constate qu'il n'est pas exclusif, puisqu'un locuteur formule 'kan tu sra gran tu fra la mèm choz' ou n ${ }^{\circ} 35$ est -ce que tu vas pouvoir sortir ?=>’èske tu poura sortir ?/ èske tu krouè $\mathrm{k}$ tu poura sortir?'

Il semble compte tenu des énoncés relevés dans Chaudenson \& Calvet (1998) que aller + verbe Inf. est d'un usage général pour l'expression du futur, soit l'exemple $\mathrm{n}^{\circ} 18: \mathrm{Ne}$ vous en faites pas, nous irons boire => 'pa avouèr pèr on va alé bouèr/ pa t'en fèr on va alé bouèr.' Ou encore le $n^{\circ} 20$ : quels fruits acheterez vous? les bananes sont bonnes. => ' ki frui $\mathrm{k}$ tu va achté ? lé fig è bone ; lé zanana é bon.'

Dans le français de Lafayette, le futur est exprimé de manière synthétique et analytique déclarent Juilland \& Conwell (1962). Le futur analytique se forme avec aller + infinitif 'on va avoir un tas des œufs'. Le futur synthétique serait favorisé par les verbes irréguliers alors que le futur analytique apparaitrait de préférence avec les verbes réguliers. Les auteurs affirment (p.179) que le futur périphrastique tend à éliminer le futur synthétique : " combined with a finite form of the verb 'aller', such phrases form periphrastic futures which tend to eliminate the synthethic in our dialect ».

\section{Dans les variétés de créoles}

Tout comme il a été procédé pour les variétés de français, nous traitons ici de la résultante du futur périphrastique français dans les franco-créoles.

Si les marqueurs du futur dans ces créoles peuvent paraitre multiples, hormis quelques occurrences éparses, de $s e^{4}$, ils constituent des allomorphes du verbe 'aller'. En effet, va et ses variantes, alle, qu'aller kay, et probablement ké sont des allomorphes du marqueur $\mathrm{du}$ futur dans ces langues. Dans le texte (A5 nº1), l'abbé Goux (1842) qui était missionnaire à la Martinique indique que le futur se forme avec 'qué' et cite des formes concurrentes telles 'qu'alle' + V ou ' $v a$ ' + V. Marie-Christine Hazaël-Massieux (2008 : 257) a effectué un comptage dans le texte du catéchisme qui a révélé quatre occurrences de $v a$, quatre qu'allé contre six qué. Un peu plus loin (p. 321), elle relève à juste titre que le marqueur du futur 'va' est peu présent dans les lettres de l'Avenir (1849) alors qu'il est 
'largement utilisé dans le créole de la bourgeoisie antillaise' : notamment chez l'abbé Goux, Marbot et Turiault. En revanche les marqueurs 'ké' et 'kalé' sont majoritaires et sont utilisés par le nègre 'créole' et 'l'arrivant', 'ke' étant davantage relevé chez le nègre 'créole' et 'kallé' chez 'l'arrivant'.

Afin de montrer les correspondances dans l'expression du futur entre les franco-créoles et les variétés de français colonial, nous avons procédé à une application de la méthode de la grammaire comparée aux langues créoles. Notre méthode d'analyse est la suivante : nous tâchons dans un premier temps de repérer les unités qui constituent le schème. Ensuite nous tentons d'identifier les formes ainsi que les schèmes puis nous faisons une mise en rapport avec des énoncés effectifs. Nous avons pris la précaution de nous référer de façon constante aux attestations de variétés de français colonial.

\section{Etablissement du schème}

\section{En antillais}

- Forme affirmative :SN + (v)a + V ou SN + (k) alé + V ou SN + ké + V

- Forme négative : $\mathrm{SN}+$ pas + ale $+\mathrm{V}$

- Marqueur préprédicatif

- Marqueur temporel

- Indique le futur

\begin{tabular}{|c|c|c|}
\hline & Formes & Attestations \\
\hline \multirow{5}{*}{ Aff. } & [a] & $\begin{array}{l}\text { A8:1849 :2/11:§ 3:yo a rété poque (ils vont rester sans rien) } \\
\text { A8:1849 :11/11§ } 4: \text { « yo a trouvé, oui encò des Chochotte, des Laroche, des Henry, } \\
\text { cétéra, cétéra » (ils vont trouver encore des Chochottes...) } \\
\text { A8 :1849:11/11§ } 5 \text { zote a vouè dévlopement là (vous allez voir le développement)! }\end{array}$ \\
\hline & [va] & $\begin{array}{l}\text { A3 :1720-40: } 1.11 \text { : mafouinque tout pas tout outi zottes va palé ça, zottes va songé } \\
\text { moé toujours (partout où vous allez parler de ça). } \\
\text { A3:1720-40: } 1.45 \text { : bientôt moé va lévé et moé va macé (bientôt je vais me lever et je } \\
\text { vais marcher). } \\
\text { A6 :1846 : ça va fè moin bien plaisi (ça va me faire bien plaisir) } \\
\text { A7 :1848 :1.77 : toé va nié moin trois fois (tu vas me nier trois fois) } \\
\text { A9:1849:1/2. § } 2: \text { li va badiner cé la qui sottes (il va badiner le sot) } \\
\text { A9 :1849:2/2. § } 3: \text { « ça ki save palé va palé » (ceux qui savent parler vont parler) }\end{array}$ \\
\hline & [(k)ale] & $\begin{array}{l}\text { A3:1720-40: } 1.64: \text { pari titaler ïo qu'alé vini quiember moé (ils vont venir me } \\
\text { chercher)? } \\
\text { A3:1720-40: } 1.61: \text { zottes pas savé cila qui pas savé veillé quiable allé tenté li (celui } \\
\text { qui ne sait pas veiller le diable va le tenter/ le tentera)? } \\
\text { A7:1848:16: z'autres qu'allé voir (vous allez voir). }\end{array}$ \\
\hline & [Ke] & A6 :1846 : yo pas ké prend moin encò (ils ne vont plus me prendre) \\
\hline & [sra] & A8 :1849:11/11§ 4 : zote sera popiété (vous serez propriétaire) \\
\hline
\end{tabular}




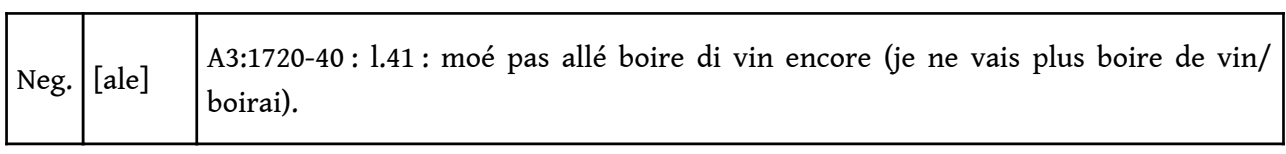

TABLEAU 1 : le futur en antillais

\section{En haïtien}

- Forme affirmative $\mathrm{SN}+(\mathrm{v}) \mathrm{a}+\mathrm{V}$ ou $\mathrm{SN}+$ ale $+\mathrm{V}$

- Forme négative $\mathrm{SN}+$ pas + alle $+\mathrm{V}$

- Marqueur préverbal

- Indicateur du futur dans l'énoncé affirmatif

La forme courte a n'apparaît que lorsqu'elle est précédée de la forme élidée des pronoms personnels sujets 1 et 2 . Dans la forme contemporaine de la langue, a est utilisé dans le contexte droit de tous les pronoms personnels, qui de ce fait adoptent la forme courte, et dans le contexte droit des substantifs. La forme longue, va, du marqueur est observée dans le contexte droit des pronoms personnels sujets à forme longue ou des SN. La forme ale peut apparaître dans un énoncé affirmatif. A la forme négative c'est exclusivement l'allomorphe ale qui apparaît dans le contexte droit de l'adverbe de négation pas.

31 Avec les prédicats statifs qui n'acceptent pas 'ap' pour exprimer l'inaccompli dans le présent, le morpheme 'ap' marque un procès au futur. Cependant 'ap' en tant que marqueur du futur n'est pas présent dans les textes sélectionnés.

\begin{tabular}{|c|c|c|}
\hline & Formes & Attestations \\
\hline Aff. & [a] & $\begin{array}{l}\text { H1 :1758 ?\$4 :ma babillé ly ben for (je vais le gronder) } \\
\text { H4/1783 : Sc1 : dré ma miré li tout proche ma babillé li ben fort (dès que je vais le } \\
\text { voir je vais le gronder) } \\
\text { H4/1783 : Sc1 : gnion nègre qui grand sorcié ma trouvé li (un grand sorcier nègre va } \\
\text { me trouver) } \\
\text { H8 : } 1796 \$ 2: \text { Si to allé Laville ta trouvé gënnecanguio (si tu vas en ville tu vas } \\
\text { trouver/:trouveras) } \\
\text { H13:1804: } 4 / 7: \text { ca to va faire quan t'a vié (qu'est ce que tu vas faire quand tu va } \\
\text { être/seras vieux)? } \\
\text { H13:1804: } 7 / 7: \text { ou t'y m'a prend l'aut' gié pour voir li (où vais-je prendre d'autres } \\
\text { yeux pour le voir?) }\end{array}$ \\
\hline
\end{tabular}




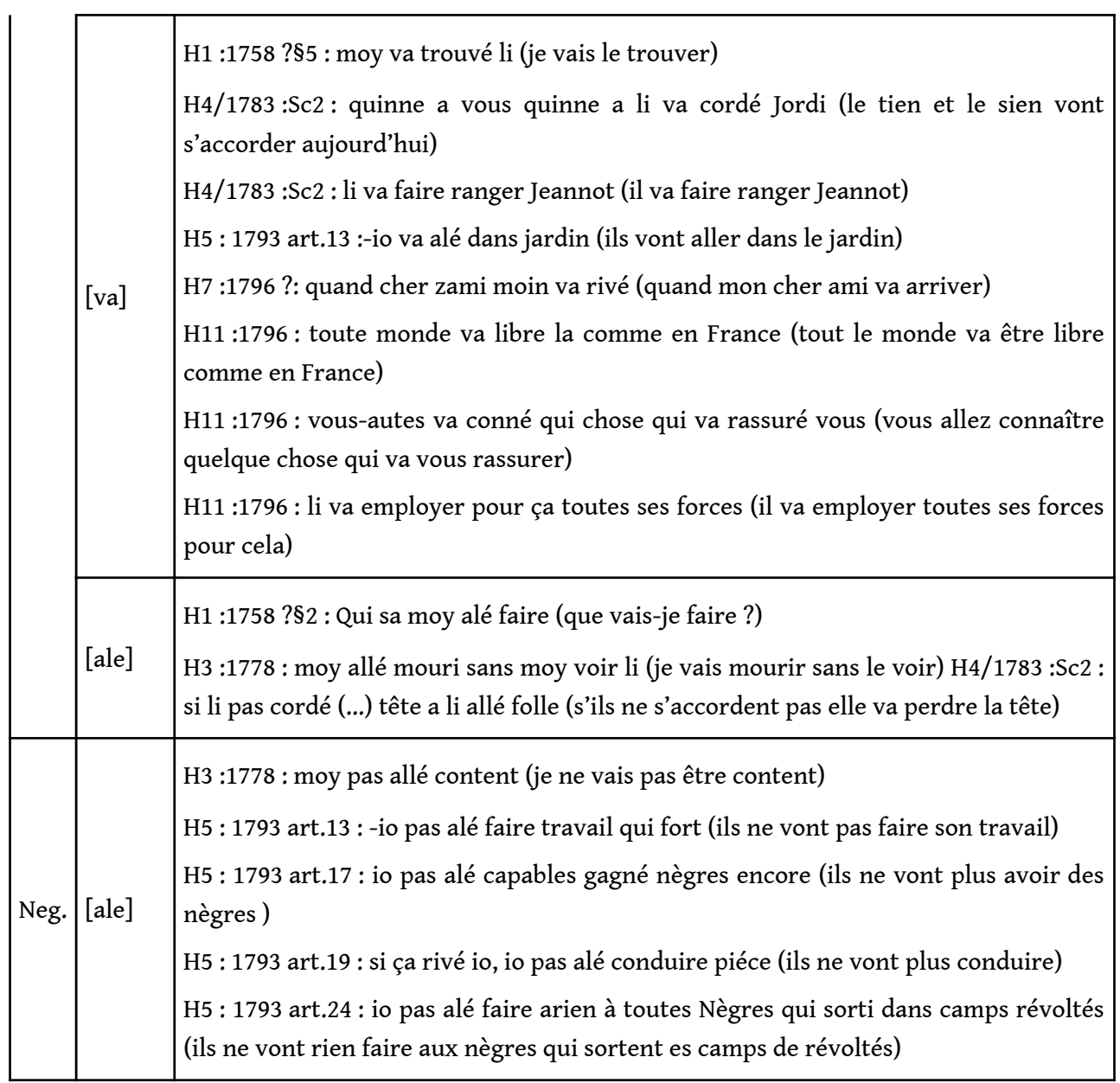

TABLEAU 2 : le futur en haïtien

Nous constatons que, dans les textes sélectionnés et sur la période sélectionnée, nous n'avons pas d'occurrence du futur avec « ap » en créole haïtien. Les marqueurs du futur à l'affirmatif sont '[a], '[va] et '[ale]. L'usage de [ale] est exclusif dans la phrase négative. Ce tableau montre que l'apparition de «ap» en tant que marqueur du futur est postérieure à la période délimitée.

\section{En louisianais}

- Forme affirmative : $\mathrm{SN}+(\mathrm{a}(\mathrm{v})) \mathrm{a}+\mathrm{V}$ ou $\mathrm{SN}+$ apé $+\mathrm{V}$ ou $\mathrm{SN}+\mathrm{sra}+\mathrm{V}$

- Forme négative : $\mathrm{SN}+\mathrm{va}+$ pas $+\mathrm{V}$

- Marqueur préverbal

- Indicateur du futur

Formes Attestations 


\begin{tabular}{|c|c|c|}
\hline & [a] & 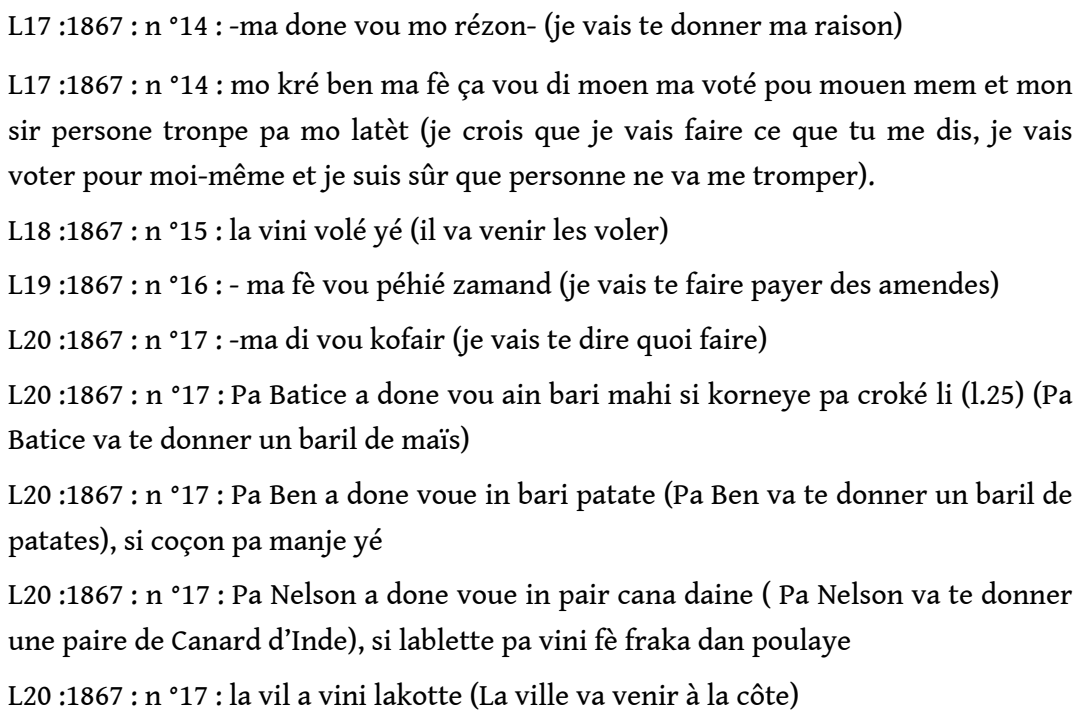 \\
\hline & [va] & $\begin{array}{l}\text { L19:1867: n }{ }^{\circ} 16: \text { paitaite yé va asté tafia avé lavian là (peut être qu'ils vont acheter } \\
\text { de la viande) } \\
\text { L19:1867: }{ }^{\circ} 16: \text { no va santé ein kantik (nous allons chanter un cantique) } \\
\text { L20:1867: n }{ }^{\circ} 17: \text { si ça rive, yé va gaigné pou nouarci Radiko (ils vont avoir à noircir } \\
\text { les Radiko) }\end{array}$ \\
\hline & [ava] & $\begin{array}{l}\text { L9 :1819: Quand patate la cuite na va mangé li, Na va mangé Na va mangé.(quand la } \\
\text { patate est cuite nous allons la manger) }\end{array}$ \\
\hline & [apé] & $\begin{array}{l}\text { L17 :1867: } n^{\circ} 14 \text { :ma pé fè dolo vini dan vou labousse pou aryen (je vais faire venir } \\
\text { de l'eau dans ta brousse pour rien) }\end{array}$ \\
\hline Neg. & $\mathrm{Va}$ & $\begin{array}{l}\text { L20 :1867 : si ça va pa vou, ma seyé pou fè ein souchripsion (si ça te va pas je vais } \\
\text { essayer de faire une souscription) }\end{array}$ \\
\hline
\end{tabular}

TABLEAU 3 : le futur en louisianais

Observons d'une part, que les marqueurs du futur sont récurrents dans les trois créoles étudiés et d'autre part quoiqu'ils paraissent nombreux, ils constituent majoritairement des allomorphes du verbe aller (ava et variantes va et a, kalé et ses variantes alé, ké). Ké apparaît assez tardivement et en nombre restreint dans le corpus sélectionné. La forme sra est mentionnée par Longin (1822), toutefois elle n'a pas été relevée d'occurrence dans nos documents, sauf dans les phrases de Longin.

Il a été également observé que dans la phrase négative le marqueur change de forme. Il devient alé en antillais et en haïtien. Pour le louisianais le corpus ne présente qu'une occurrence de négation en va. Toutefois le phénomène de changement de forme du marqueur du futur dans la phrase négative est attesté en Louisianais par NeumannHolzschuh (1987) et Klingler (2003).

\section{En français colonial}

Dans les variétés de français constituant notre corpus français, le futur est rendu sous diverses formes. A l'affirmative il est exprimé dans nombre de phrases à la première et 
seconde personne du singulier par : m'a $\mathrm{V}$ inf. / t'a+V inf. D'autres structures comme $\mathrm{SN}+\left\{\left(s^{\prime} e n\right) v a\right\}$ ou $\mathrm{SN}+\{a p e ́\}+\mathrm{V}$ inf. ou $\mathrm{SN}+\{$ sre $\}+\mathrm{Inf}$. ont été aussi relevées.

\begin{tabular}{|c|c|c|}
\hline & Formes & Attestations \\
\hline & [a] & $\begin{array}{l}\text { Queb.Dör : m'a y aller } \\
\text { Mis.W.A.D.115 : « ta voler mon cheval, } \\
\text { Mis.WA D } 118: \text { Ma aller kri l'aut } \\
\text { Mis.WAD.116 : « mouè ma cogner ! } \\
\text { Mis.M.C. : 'quocé t'as prend', la tête ou ben les racines?' }\end{array}$ \\
\hline & [va] & 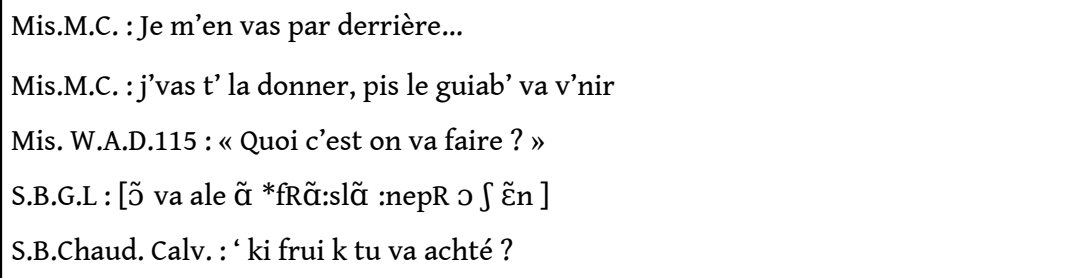 \\
\hline & [ava] & Mis.WARD115 : Belle de Jour a'va vous pagner tous les jours. Belle Finette a'va balier. \\
\hline & [apé] & $\begin{array}{l}\text { Mis.WARD115: quand t'es après pagner la vieille, ma t'apporter la hache, pi tu } \\
\text { pourras lui couper son cou. }\end{array}$ \\
\hline & [sre] & S.B.Chaud.Calv. : Amik $t$ ariv j srè peutèt kimanj \\
\hline Neg. & Irai & $\begin{array}{l}\text { Queb.Dör : j'irai pas } \\
\text { Mis. «J'vas pas». }\end{array}$ \\
\hline
\end{tabular}

\section{Tableau 4 : LE FUTUR EN FRANÇAIS COLONIAL}

\section{Mise en évidence des correspondances dans les créoles haïtien, antillais et louisianais}

\begin{tabular}{|c|c|c|c|c|}
\hline Formes & Français col. & Haïtien & Antillais & Louisianais \\
\hline [a] & $\begin{array}{l}\text { mouè ma } \\
\text { cogner }\end{array}$ & $\begin{array}{l}1758 \text { ?\$4 : ma babillé ly ben } \\
\text { for }\end{array}$ & $\begin{array}{l}1849: 2 / 11: \S 3: \text { yo } \\
\text { a rété poque }\end{array}$ & $\begin{array}{l}1867: \mathrm{n}{ }^{\circ} 16:- \\
\text { ma fè vou péhié } \\
\text { zamand }\end{array}$ \\
\hline [va] & $\begin{array}{l}\text { j'vas t' la } \\
\text { donner, }\end{array}$ & 1758 : moy va trouvé li & 1720 : moé va lévé. & $\begin{array}{l}1867: \mathrm{n}^{\circ} 16: \text { no } \\
\text { va santé ein } \\
\text { kantik }\end{array}$ \\
\hline [ava] & $\begin{array}{ll}\text { Belle de } & \text { Jour } \\
\text { a'va } & \text { vous } \\
\text { pagner } & \end{array}$ & $\begin{array}{l}\text { [Ava] existe dans cette } \\
\text { fonction en haïten mais } \\
\text { nous n'avons } \\
\text { d'attestations dans les } \\
\text { textes sélectionnés }\end{array}$ & $\begin{array}{l}\text { Nous n'avons } \\
\text { aucune attestation } \\
\text { de ce marqueur } \\
\text { pour cette variété. }\end{array}$ & $\begin{array}{l}\text { L9: } 1819: \text { Quand } \\
\text { patate la cuite na } \\
\text { va mangé li }\end{array}$ \\
\hline
\end{tabular}




\begin{tabular}{|c|c|c|c|c|c|}
\hline & [apé] & $\begin{array}{l}\text { Observé très } \\
\text { rarement } \\
\text { Cf. tableau } \\
\text { précédent }\end{array}$ & $\begin{array}{l}\text { [ape] existe dans cette } \\
\text { fonction en haïtien } \\
\text { contemporain mais nous } \\
\text { n'avons pas d'attestations } \\
\text { dans les textes sélectionnés }\end{array}$ & $\begin{array}{l}\text { Nous n'avons } \\
\text { aucune attestation } \\
\text { de ce marqueur } \\
\text { pour cette variété. }\end{array}$ & $\begin{array}{l}1867: \mathrm{n}{ }^{\circ} 14: \text { ma } \\
\text { pé fè dolo vini } \\
\text { dan vou labousse } \\
\text { pou aryen. }\end{array}$ \\
\hline & [sre] & $\begin{array}{l}\text { Marqueur du } \\
\text { futur connu } \\
\text { en français }\end{array}$ & & & \\
\hline & $\begin{array}{l}{[(\mathrm{k})} \\
(\mathrm{al}) \mathrm{e}]\end{array}$ & $\begin{array}{l}\text { Marqueur } \\
\text { connu en } \\
\text { français sous } \\
\text { la forme [ale] }\end{array}$ & & $\begin{array}{l}\text { A3:1720-40: } 1.64: \\
\text { pari titaler io } \\
\text { qu'alé } \\
\text { quiember moé? }\end{array}$ & \\
\hline Neg. & $\begin{array}{l}\text { [ire] } \\
{[\text { va] }} \\
\text { [ale] }\end{array}$ & \begin{tabular}{ll|} 
Marqueur & \\
connu & en \\
français &
\end{tabular} & $\begin{array}{l}\text { H3 :1778: moy pas allé } \\
\text { content }\end{array}$ & $\begin{array}{l}\text { A3 : 1720-40: } 1.41: \\
\text { moé pas allé boire } \\
\text { di vin encore. }\end{array}$ & $\begin{array}{l}\text { L20:1867 : si ça } \\
\text { va pa vou, }\end{array}$ \\
\hline
\end{tabular}

\section{Tableau 5 : LE FUTUR : CORRESPONDANCES}

Selon nos observations, le prospectif est rendu dans la grande majorité des occurrences relevées dans les créoles français par les marqueurs : va ou ses variantes ava, $a$, ou sra ou sera ou ale ou ses variantes aprale, kale ou kay mais cet aspect est également rendu par le biais de stratégies telle l'utilisation des adverbes et locutions adverbiales. Véronique (2006) étudie l'émergence de la référence temporelle dans les langues créoles et relève que les verbes thématiques par exemple fini, fonctionnent comme marqueurs préverbaux précoces dans plusieurs créoles français (haïtien, guadeloupéen, mauricien); de même, l'usage des adverbiaux est également relevé comme moyen d'expression de la temporalité. Il semble toutefois qu'il n'y ait pas d'homogénéité dans l'apparition des marqueurs préverbaux au sein des créoles français. Ils apparaissent après environ un siècle de colonisation en Haïti mais une vingtaine d'années à Maurice.

\section{Conclusion}

Notre objectif a été de montrer l'implication des variétés de français colonial dans le développement du marqueur du futur dans les franco-créoles. Nous avons à cette fin appliqué la méthode de la grammaire comparée aux langues créoles. Nous avons expliqué les raisons principales en cause dans la préférence pour la forme périphrastique du futur dans le moyen français. Cette préférence a été confirmée dans les variétés de français colonial et constitue principalement le marqueur du futur dans les franco-créoles.

Puisque va est attesté en tant que marqueur du futur en français et dans les créoles français, est-il un marqueur français ou créole? Cette question pertinente a été posée par Guy Hazaël-Massieux (1996) tenant compte du fait que le futur en va est présent dans l'ensemble des créoles français mais également au Canada. Il y reconnaît une forme encore bien française : 
"Quand la créolisation commence-t-elle vraiment? Nous pouvons supposer que l'emploi de va est encore assez français puisque nous le retrouvons non seulement dans la Caraïbe, la Guyane, la Louisiane et l'océan Indien, mais même au Canada où, semble-il la forme de $1^{\text {re }}$ personne 'm'a venir' apparaît comme à Saint-Thomas ou Haïti, mais avec les mêmes contraintes que l'on retrouvera à la Réunion sur l'emploi d'un futur synthétique à la forme négative ».

\section{BIBLIOGRAPHIE}

Bauche, H. (1946). Le langage populaire, Paris: Ed. Payot.

Brunot, F. (1935). Histoire de la langue française des origines à 1900, T. VIII fasc.2, p. 1117-1147.

Chaudenson, R. (2003). La créolisation : théorie, applications, implications, Paris: L'Harmattan.

Chaudenson, R. (1995b). « Les français d'Amérique ou le français des Amériques ? » : Genèse et comparaison, dans : R. Fournier et H. Wittmann (éds.), Le français des Amériques, p. 3-19.

Chaudenson, R. (1995a). De la Vendée aux Caraïbes. Le Journal d'André Massé, Etudes Créoles XVIII, 2, p. 97-110.

Chaudenson, R. (1994). «Les créoles français sont-ils des formes simplifiées du français ? ", dans : F. Lapeyre (éd.), «Simple-simplification », Cahiers du français contemporain, décembre n¹, p. 41-54.

Chaudenson, R. (1979). Les créoles français. Paris: Nathan.

Degraff, M. (1999). Language creation and language change: Creolization, diachrony and development, Cambridge, MA: MIT Press.

Degraff, M. (2001). « Morphology in Creole genesis: Linguistics and ideology », dans : M.

Kenstowicz (éd.), Ken Hale: A life in language, Cambridge, MA: MIT Press, p. 53-121.

Degraff, M. (2001). On the origin of Creoles: A Cartesian critique of 'Neo'-Darwinian linguistics, Linguistic Typology 5, 2, p. 213-310.

Degraff, M. (2003). Against creole exceptionalism. Discussion note. Language 79, p. 391-410.

Dörper S. (1990). Recherches sur ma + infinitif 'je vais' en français, Revue québécoise de linguistique 19/1, p. 101-127. Consulté le 17/02/2011 sur site : http://id.erudit.org/iderudit/602667ar.

Dyen, I., Kruskal J. \& Black, P. (1992). An Indo-European Classification: A lexicostatistical Experiment, American Philosophical Society, 82/5

Fattier, D. (1998). Contribution à l'étude de la genèse d'un créole: l'Atlas Linguistique d'Haïti, cartes et commentaires, thèse de doctorat d'état, Université de Provence, T. 1-5

Fennell, T.G. (1975). La morphologie du Futur en Moyen Français. Genève: Droz

Galdi, L. (1949). « De l'importance des parlers français-créoles pour la linguistique

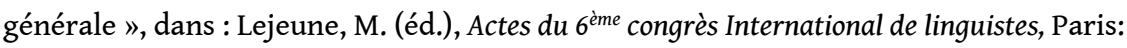
Klincksieck. 
Goyette, S. (2000). « From Latin to early Romance, a case of partial Creolization? », dans : McWhorter, J. (éd.), Language change and language contact in Pidgins and Creoles, p. 103-131.

Hagège C. (1985). L'homme de paroles. Paris: Fayard.

Hazaël-Massieux, G. (1996). Les créoles : problèmes de genèse et de description. Presses de l'Université de Provence.

Hazaël-Massieux, M.-C. (2005). Théories de la genèse ou histoire des créoles : l'exemple du développement des créoles de la caraïbe. La linguistique, Revue de la Société Internationale de Linguistique Fonctionnelle. Les créoles. Vol. 41-1, P.U.F.

Hazaël-Massieux M.-C. (2008). Textes anciens en créole français de la Caraibe : Histoire et analyse, Paris: Publibook.

Jourdain, E. (1956). Du français aux parlers créoles. Paris: Klincksieck.

Manessy, G. (1995). Créoles, pidgins, variétés véhiculaires. Procès et genèse, CNRS édition.

Meillet, A. (1948). Linguistique historique et linguistique générale. Paris: Edouard Champion.

Mufwene, S. (1986). Les langues créoles peuvent-elles être définies sans allusion à leur histoire?, Etudes créoles, vol. IX, $n^{\circ}$ 1, p. 135-150.

Mufwene, S. (1993). Africanisms in Afro-American Languages Varieties, Athens: The University of Georgia Press.

Mufwene, S. (2005). Créoles, écologie sociale, évolution linguistique. Paris: L’Harmattan.

Mufwene, S. (2002). Typologie des définitions des créoles, Linguistique et créolistique. Univers créoles 2, Paris: Economica.

Mufwene, S. (2007). Les créoles : de nouvelles variétés indo-européennes désavouées ?, Actes du colloque : Créolisation linguistique et sciences humaines, Paris: Presses Universitaires HatianoAntillaises.

Picoche, J. \& Marchello-Nizia, Ch. (1989). Histoire de la langue française, Paris: Nathan, ( $3^{\text {ème éd. }}$ 1994).

Sundell, L-G. (1991). Le temps futur en français moderne, Stockholm: UPPSALA

Thomason, S. G. \& Kaufman, T. (1988). Language contact, Creolization, and Genetic linguistics, Berkeley : University of California.

Valdman, A. (1976). Certains aspects sociolinguistiques des parlers créoles aux Antilles, Ethnies, $n^{\circ}$ 3, p. 7-22.

Valdman, A. (1978) Le créole : structure statut et origine. Paris: Klincksieck.

Véronique, D.(2006). « Trois aspects de la temporalité dans les créoles français : Aktionsart, référence au passé et finitude », 23/03/06, Paris: SFL UMR7023.

Consulté le 12/01/2016 sur site : http://www.umr7023.cnrs.fr/sites/sfl/IMG/pdf/veronique. 27.03.06.handout.pdf

Véronique, D. (2007). Ce que les langues créoles nous enseignent à propos de la genèse des langues, Actes du colloque : Créolisation linguistique et sciences humaines, Paris: Presses Universitaires Hatiano-Antillaises. 


\section{ANNEXES}

\section{CORPUS DE TEXTES}

\section{Liste des textes et documents consultés pour les variétés de français}

Concernant les variétés de français colonial, nous avons eu recours à plusieurs auteurs et ouvrages et fournissons ci-dessous la liste des documents et textes sur lesquels nous nous sommes arrêtés pour nos analyses. Il s'agit de :

\section{De Saint-Barthélemy}

Lefebvre, G. (1976). «Français régional et créole à Saint-Barthélemy », dans : Valdman \& Snyder (éds.), Actes du premier colloque sur l'identité culturelle et la francophonie dans les Amériques, Québec, Presses de l'Université Laval.

Calvet, L.-J. \& Chaudenson, R. (1998). Saint Barthélemy : une énigme linguistique. Paris: Didier Erudition, Collection Langues et développement.

\section{Du Missouri (old Mines \& Sainte Geneviève)}

Carrière, J. M. (1937). Tales from the French Folk-lore of Missouri, Evanston and Chicago: Northwestern University.

L'auteur a pu réunir ces contes grâce à ces deux conteurs $\mathrm{M}$. Joseph Ben Coleman et $\mathrm{M}$. Franck Bourisaw respectivement âgés de 40 et 70 ans à l'époque de la collecte.

Nous avons parcouru l'ouvrage dans son ensemble et nous nous sommes arrêtés sur un total de 13 contes $\left(\mathrm{N}^{\circ} 1,2,9,21,52,53,55,56,61,62,37,25,7\right)$ pour des analyses plus approfondies. Cette dizaine de contes constitue plus de $10 \%$ des contes et a été sélectionnée à travers tous les types définis par l'auteur.

Miller, W. M. (1930). Missouri's paw -paw French, The French Review, Vol. 3, No. 3 (Jan., 1930), p. 174-178.

Ward, A. D. (1935). The Survival of French in the old district of Sainte Geneviève, A quarterly of research, Vol. X, No. 2, The University of Missouri Studies.

\section{De Frenchville}

Mougeon, F. \& Uritescu, D. (2006). Les derniers français de Frenchville, La Linguistique, vol. 42, fasc. 1, p. 115-128.

Caujolle, J. (1972). « Esquisse d'une description du parler français de Frenchville, Pennsylvanie ", dans : The French Language in the Americas (Newsletter of the French VIII Section of the MLA), 16, p. 26-32.

\section{De la Louisiane francophone}

Conwell, M.J. \& Juilland, A. (1963). Louisiana french grammar, The Hague: Mouton \& Co. Pellerin, E. (1950). La langue française en Louisiane, thèse de doctorat : Département de français de l'université de Mc Gill. 


\section{Liste des textes et documents consultés pour les variétés de créole}

En ce qui concerne les documents relatifs aux langues créoles nous avons fait usage, sans nous y limiter, en grande partie des textes anciens publiés par Marie-Christine Hazaël-Massieux (2008) pour les variétés de la Caraïbe. Nous avons également eu recours pour les variétés de la Louisiane à Klingler (2003), Neumann-Holzschuh (1988), Allen, Ware, \& Garrison Slave song (1867), Georges W. Cable (1886) The dance in place Congo. Aussi nous avons pu consulter quelques documents plus rares et difficiles d'accès que sont The Mermaid de Lieber gardé à la South Carolina Library et le Manuel des habitants de Saint-Domingue de Ducœurjoly gardé à l'Institut de France. Pour les documents relatifs aux différentes variétés de français, nous avons pu gracieusement bénéficier du corpus des deux octogénaires qui a été mis à notre disposition par Françoise Mougeon, Edouard Mougeon et Edouard Beniak.

\section{NOTES}

1. T. 1. P. 52

2. It must be recognized that there is no reason whatsoever for excluding the possibility that Latin, to some degree at least, was creolized; indeed when comparing its external history to that of other European languages, it must be conceded that creolization is far likelier to have played a role in the history of Latin/Romance than in that of any other language, p. 6.

3. Cf. Corpus de textes p.23.

4. Longin 1822 : A4. $n^{\circ} 1$ : fait état de sra et sré comme des marqueurs respectifs du futur et du Conditionnel.

\section{RÉSUMÉS}

Les dernières décennies ont connu en linguistique créolistique un débat intense opposant les tenants de la position stipulant le développement des langues créoles comme anormal (Thomason \& Kaufman 1988, I. Dyen, J. Kruskal \& P. Black 1992) et ceux considérant la naissance des langues créoles comme non-exceptionnelle (Michel Degraff 2003, Mufwene 2007). Au nombre des éléments présentés pour avancer le classement des langues créoles dans 'une catégorie à part' sont cités les marqueurs préverbaux. En effet, ceux-ci sont perçus comme faisant partie des éléments les plus divergents de la langue européenne, de par leurs formes actuelles ainsi que leur fonctionnement plutôt analytique. Pourtant, comme nous le verrons dans notre réflexion qui s'intéresse tout particulièrement aux créoles français et à l'expression du futur dans ces idiomes, l'étymologie française est indubitable. Elle se confirme à travers l'étude des formes, du sémantisme, et du fonctionnement syntaxique.

Le caractère analytique du futur créole vient de la préférence pour la périphrase verbale (aller+infinitif) compte tenu des difficultés engendrées par le manque d'analogie du futur synthétique mais également parce que les formes analytiques, le dit Meillet (1948), permettent une meilleure expressivité. Ainsi, quoique le futur ait pris différentes formes dans les créoles par 
la suite, la première forme du marqueur ((v)a) est attestée tout aussi bien dans les français d'Amérique que dans les créoles français et les formes concurrentes sont tout aussi françaises (sra, ale, ap).

Creole languages are the most recent natural languages (Khim 2005). As a matter of fact the question of their classification imposed itself very early to linguists when the language science enquired about the topic of language filiation in the second half of $19^{\text {th }}$ Century. In the same century, some linguists qualified these languages as "mixed". Mixed like the population that used to speak them. Others in the $20^{\text {th }}$ century (Thomason \& Kaufman 1988) described them as a category of language with no genesis for they were like linguistic monsters. Indeed not resembling one's parents is a kind of monstrosity, Aristotle says.

This article deals with historical comparative research. It aims at bringing a contribution to the establishment of relatedness between three French-based Creoles (Haitian, Antillean (Guadeloupe/Martinique) Louisianan and the varieties of colonial French. The colonial French documents are from St. Barthelemy, Missouri, Frenchville, Francophone Louisiana and Quebec. The predicative syntagm of the three Creoles are compared on the one hand and on the other hand a comparison is established with the varieties of colonial French. The corpus is composed of two kinds of texts: the texts showing the early stage of the Creole languages and the documents showing the language state of Colonial French. The research mainly tackles the TMA system and deals with the impact of French periphrastic future in the development of French based Creoles. In specialized literature, the filiation of Creole languages is subject to debate. Because of their status of contact language they are usually designated as non-normal (Thomason \& Kaufman 1988, Dyen, Kruskal \& Black 1992). Other scholars consider their birth as non-exceptional (Degraff 2003, Mufwene 2007). Among the items mentioned to classify Creole languages as nonnormal languages, we find the TMA markers. They are perceived as one of the most divergent items from the European language because of their present form and analytical functioning. However, as we will try to show it, the expression of future in French based Creoles has undoubtedly a French origin. This origin is confirmed through the study of the forms, the meaning and the syntactic functioning.

Thanks to the study of a corpus of texts presenting colonial varieties of French, but also ancient texts in Haitian, Antillean, and Louisiana Creoles dated respectively from 1671 to 1850 and 1867 we intend to show the impact of colonial varieties of French in the development of French based Creoles.

We proceed as follows: first, we intend to understand how and why periphrastic future was preferred by the French settlers. Then we introduce the results of the analyses of both Colonial French and Creole corpus. At last we put forward the corresponding patterns between the two groups of languages.

INDEX

Keywords : colonial French, Creole, Haiti, Antillies, Louisiana, Historical Comparative

Mots-clés : français colonial, créole, Haïti, Antilles, Louisiane, Grammaire Comparée

\section{AUTEUR}

MARIE E. PAUL

Centre Universitaire Catholique du Bourgogne

Paris III Sorbonne Nouvelle 
magensie@yahoo.fr 